\title{
Dynamic Modeling and Operation Characteristics Analysis of LiBr Absorption Heat Pump
}

\author{
*Hu Xianfa, Zhang Shuqing, Peng Zhen, Tang Shaopu, Liu Ning \\ Department of Electrical Engineering, Tsinghua University ,Beijing , 100084, china;
}

\begin{abstract}
In order to fully exploit the dynamic operation characteristics of absorption heat pump, a dynamic simulation model of $\mathrm{LiBr}$ absorption heat pump considering mass transfer and distribution parameters is established. The thermal inertia characteristics of the system are studied, and the thermal performance and crystallization characteristics of the system are analyzed under the conditions of heat source temperature change, cooling water temperature change and refrigerant water temperature change. The results show that: the model can accurately simulate the steady-state and dynamic characteristics of the absorption heat pump; the thermal inertia of the unit is mainly related to the mass of the solution stored in the unit; the upper limit of the heat source inlet temperature is affected by the COP of the system and the crystallization risk, the decrease of the inlet temperature of cooling water can increase the COP of the system, and the lower limit is limited by the crystallization risk, and the cooling water inlet temperature is affected by the crystallization risk, and the lower limit of the inlet temperature of cooling water is limited by the crystallization characteristics. The upper limit of the inlet temperature of refrigerant water is not limited by the crystallization characteristics, but is mainly limited by the user's energy demand. This provides a reference for the dynamic operation of absorption heat pump.
\end{abstract}

\section{Introduction}

In order to improve energy efficiency and reduce pollutant emissions, integrated energy system has been developed rapidly in China in recent years. There are a lot of waste heat and waste heat of different temperature levels in the integrated energy system of Industrial Park, and the energy supply form is different. Driven by heat energy, absorption heat pump with the characteristics of low power consumption, waste heat reuse and flexible operation method plays an increasingly important role in the integrated energy system. Because the energy supply system is close to the user end, the cooling and heat load of the user side will fluctuate greatly with the change of the ambient temperature, the uncertainty of the user behavior and other factors, which leads to the constant change of the operating conditions of the absorption heat pump. The dynamic characteristics and control characteristics of absorption heat pump are studied by dynamic simulation, which can provide important technical support for the dynamic operation control of absorption heat pump in integrated energy system.

At present, absorption heat pump modeling is mainly based on the conservation of mass, momentum and energy. Jeong [1] established the dynamic model of absorption heat pump and studied the control equations of the parameters such as heat exchange area of each component and solution circulation volume on the thermal performance of the system. The simulation calculation is complex and can not meet the real-time requirements of the system simulation. Kohlenbacha ${ }^{[2,3]}$ studied the influence of thermal inertia on the dynamic characteristics of the refrigerator, and showed that the dynamic response time of the system increased with the increase of thermal inertia, but did not make further research on its thermal performance. Khalid $\mathrm{A}^{[4]}$ focuses on the description of static characteristics of various components of absorption heat pump, especially the absorber components, which can not reflect the dynamic characteristics of the system under external disturbance. Naoki Sugan ${ }^{[5]}$ established the dynamic parameter model of single effect unit, and used distributed parameter treatment for the solution heat exchanger and generator with long solution residence time, so the calculation was complex. To sum up, the dynamic modeling of absorption heat pump needs to focus on the heat and mass transfer effect of absorber, the heat storage effect of heat exchanger, generator, absorber and other equipment components, focus on the influence of heat storage inertia on the dynamic characteristics of absorption heat pump, further study the performance characteristics and crystallization characteristics of the unit when the external conditions change, and obtain the rules of safe and efficient operation of the system ,This is of great significance to the dynamic optimal regulation of the system in actual operation.

In this paper, the dynamic model of absorption heat pump is established, in which the heat and mass transfer model based on Nusselt theory ${ }^{[6]}$ is adopted for the absorber,

*Email:gminerwolf@163.com; 
taking into account the accuracy and calculation efficiency. The lumped parameter dynamic model is adopted for other equipment, fully considering the energy storage effect of the equipment. The test results show that the model has good accuracy under steady-state and dynamic conditions. Based on the model, the factors affecting the thermal inertia of the system are further studied, and the thermal performance and crystallization characteristics of the unit under off design conditions are analyzed, which has a certain reference value for the dynamic operation and regulation of the absorption heat pump in the integrated energy system.

\section{Principle and process of absorption heat pump}

The structure of single effect $\mathrm{LiBr}$ absorption heat pump is shown in Figure 1. The main components include generator, absorber, evaporator, condenser, solution circulating pump, throttle valve, etc. The driving heat source fluid (flue gas, steam, hot water, etc.) releases heat to lithium bromide solution through the generator to generate refrigerant steam; the steam is condensed into saturated water by circulating cooling water through the condenser, and the condensed water enters the low-pressure evaporator through the throttle valve; the condensed water in the evaporator changes into steam through heat exchange with the refrigerant water; the refrigerant steam in the evaporator is absorbed by lithium bromide solution in the absorber And heat exchange to the absorber; The cooling circulating water first enters the absorber to cool it to maintain the temperature of $\mathrm{LiBr}$ solution in the absorber, and then cools the steam in the generator through the condenser to maintain the back pressure in the condenser to ensure the circulation of working medium.

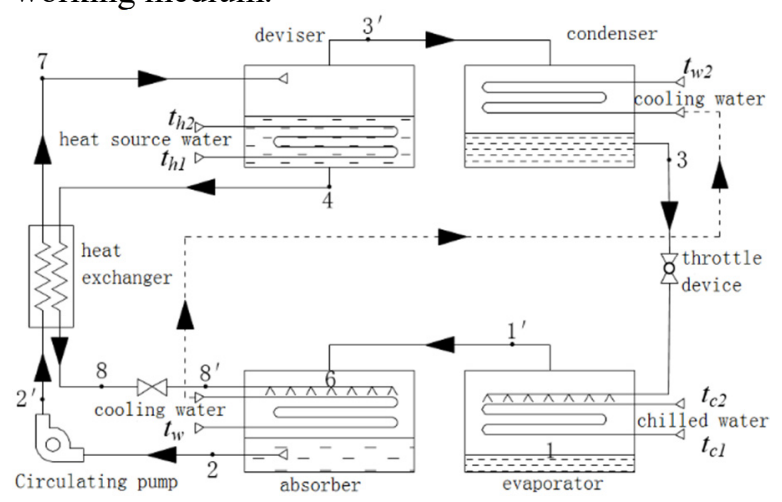

Fig 1 Schematic diagram of $\mathrm{LiBr}$ absorption heat pump

\section{Modeling of absorption heat pump}

In order to simplify the model and facilitate the calculation, it is necessary to make assumptions on the model: the physical property parameters of the solution in each container are treated as lumped parameters; the heat transfer along the length of the heat exchange tube is not considered; each fluid flows in one-dimensional homogeneous flow along the flow direction and is treated with equal cross-section; the heating quantity entering the generator is used for solution heating completely, and the solution outlet of the absorber is in equilibrium; the change of flow rate caused by the temperature of concentrated solution side and the change of solution density and specific heat at the inlet and outlet are not considered.

\subsection{Model of generator}

There are gas-liquid two phases in the generator due to the evaporation of circulating working medium. For two-phase fluid, the mass conservation equation, volume conservation equation, solute mass conservation equation, energy conservation and other control equations are established, as shown in equation (1-5).

$$
\begin{aligned}
& \frac{d M_{l, f}}{d t}=m_{l, \text { in }, f}-m_{l, \text { out }, f}-m_{v, f} \\
& \frac{d M_{g, f}}{d t}=m_{v, f}-m_{v, \text { out }} \\
& V_{f}=\frac{M_{g, f}}{\rho_{g, f}}+\frac{M_{l, f}}{\rho_{l, f}} \\
& M_{l, f} \frac{d \xi_{f}}{d t}+\xi_{f} \frac{d M_{l, f}}{d t}= \\
& \quad m_{l, \text { in }, f} \xi_{l, \text { in }, f}-m_{l, \text { out }, f} \xi_{f} \\
& \frac{d\left(M_{l, f} c_{f} T_{f}\right)}{d t}=m_{l, \text { in }, f} h_{l, \text { in }, f} \\
& -m_{l, \text { out }, f} h_{l, \text { out }, f}-m_{v, f} h_{v, f}+Q_{f}
\end{aligned}
$$

Where $M_{l, f}$ is the mass of solution in the generator and $M_{g, f}$ is the mass steam in the generator, $\mathrm{kg} ; m_{i, \text { in, } f} 、 m_{i, \text { out }, f}$ are the mass flow rate of solution at the inlet and outlet of the generator, $\mathrm{kg} / \mathrm{s} ; m_{v, f}$ is the evaporation capacity of solution in the generator, $\mathrm{kg} / \mathrm{s} ; m_{v, \text { out }}$ is the steam flow rate at the outlet of the generator, $\mathrm{kg} / \mathrm{s} ; V_{f}$ is the volume of the generator, $\mathrm{m}^{3} ; \rho_{l, f}, \rho_{g, f}$ are the density of solution and steam, $\mathrm{kg} / \mathrm{m} 3 ; \xi_{l, i n, f}$ and $\xi_{, f}$ are the concentration of solution at the inlet and outlet of the generator, $\% ; h_{l, i n, f}$ is the enthalpy of solution at generator inlet, $h_{l, o u t, f}$ is the enthalpy of solution at generator outlet and $h_{v, f}$ is the enthalpy of steam at generator outlet, $\mathrm{KJ} / \mathrm{Kg}$; $c_{f}$ is the specific heat of lithium bromide solution, $\mathrm{kJ} /(\mathrm{kg} \cdot \mathrm{K}) ; T_{f}$ is the temperature of lithium bromide solution, ${ }^{\circ} \mathrm{C} ; Q_{f}$ is the heat exchange power of generator and driving heat source medium, $\mathrm{kW}$, The calculation formula is shown in equation (6):

$$
\left\{\begin{array}{c}
U=U_{0}\left(\frac{m_{q}}{m_{q 0}}\right)^{0.8} \\
Q_{f}=\frac{U A\left(\Delta T_{\max }-\Delta T_{\min }\right)}{\ln \frac{\Delta T_{\max }}{\Delta T_{\min }}}
\end{array}\right.
$$

Where, $U_{0}$ is the heat transfer coefficient under rated condition, $m_{q 0}$ is the mass flow rate of heat source medium, $\mathrm{kg} / \mathrm{s} ; A$ is the heat exchange area, $\mathrm{m}^{2}$. 


\subsection{Model of absorber}

In the process of absorbing water vapor by $\mathrm{LiBr}$ aqueous solution in the absorber, it is assumed that the establishment speed of temperature field on the surface of liquid film is much faster than that of concentration field, and the sensible heat of water vapor release is ignored, then the heat and mass transfer of water vapor absorbed by solution can be divided into two independent processes, and the calculation decoupling of heat and mass transfer is completed ${ }^{[7]}$. The formula for calculating the mass flow rate of water vapor absorbed by the inner tube bundle surface of the absorber is shown in equation (7). The lithium bromide solution in the absorber absorbs the heat of steam and also releases heat to the circulating cooling water. The formula for calculating the heat release power of cooling water ${ }^{[7]}$ is shown in equation (8).

$$
\left\{\begin{array}{c}
K=0.918 \frac{D^{0.5} g^{1 / 6} \tau_{s}^{1 / 3} \rho_{l}^{5 / 6}}{d_{0}^{1 / 6} v_{l}^{1 / 6}} \\
m_{a b}=K A_{a b}\left(\xi_{a b, \text { in }}-\xi_{\text {int }}\right)
\end{array}\right.
$$

Where, $m_{a b}$ is the absorption capacity of water vapor per unit time, $\mathrm{kg} / \mathrm{s} ; A_{a b}$ is the surface area of the solution in contact with the vapor, $\mathrm{m}^{2}, \xi_{a b, \text { in }}$ and $\xi_{\text {int }}$ are the solution concentration in the absorber solution and at the gas-liquid junction respectively, $\% ; K$ is the mass transfer coefficient characterizing the adsorption capacity of the solution; $D$ is the diffusion coefficient; $\tau_{s}$ is the mass flow rate of lithium bromide solution per unit tube length, $\mathrm{kg} / \mathrm{s} ; \rho_{l}$ is the density of lithium bromide solution at the gas-liquid junction, $\mathrm{kg} / \mathrm{m}^{3} ; v_{l}$ is the dynamic viscosity, $\mathrm{N} \cdot \mathrm{s} / \mathrm{m}^{2}$.

$$
\left\{\begin{array}{c}
\alpha_{x s}=\left(\frac{\lambda_{l}^{3} g \rho_{l}^{2}}{3 \tau_{s} v_{l}}\right)^{\frac{1}{3}} \\
Q_{x s}=\alpha_{x s} A_{x s}\left(T_{i n}-T_{x s, c w}\right)
\end{array}\right.
$$

Where, $\lambda_{l}$ is the heat conductivity at the interface of liquid-liquid of lithium bromide solution, $T_{\text {in }}$ and $T_{x s, c w}$ are the temperature at the gas-liquid junction of lithium bromide solution in the absorber and the average temperature of the cooling water absorbed in the absorber, ${ }^{\circ} \mathrm{C} ; \alpha_{x s}$ is the heat exchange coefficient of the absorber.

\subsection{Model of evaporator and condenser}

Due to the phase change process of a single working medium in the evaporator and condenser, The governing equations mainly include mass conservation equation, solvent conservation equation and energy conservation equation. Taking the evaporator as an example, the governing equation is expressed as equation (9):

$$
\left\{\begin{array}{c}
\frac{d M_{l, z}}{d t}=m_{i n, z}-m_{v, z} \\
\frac{d M_{g, z}}{d t}=m_{v, z}-m_{\text {out }, z} \\
\frac{M_{l, z}}{\rho_{l, z}}+\frac{M_{g, z}}{\rho_{g, z}}=V_{z} \\
\frac{d\left(M_{l, z} c_{z} T_{z}\right)}{d t}=m_{\text {in }, z} h_{\text {in }, z}+Q_{z}-m_{\text {out }, z} h_{\text {out }, z}
\end{array}\right.
$$

Where, $m_{i n, z}$ is the water flow into the evaporator, $\mathrm{kg} / \mathrm{s}$; $m_{v, z}$ is the evaporation capacity of water vapor in the evaporator, $\mathrm{kg} / \mathrm{s} ; m_{\text {out }, z}$ is the steam flow at the outlet of the evaporator, $\mathrm{kg} / \mathrm{s} ; M_{l, z}$ and $M_{g, z}$ is the mass of liquid and gas in the evaporator, $\mathrm{kg} ; \rho_{l, z}$ and $\rho_{g, z}$ are the density of liquid and gas in the evaporator, $\mathrm{kg} / \mathrm{m}^{3} ; V_{z}$ is the volume of the evaporator, $\mathrm{m}^{3} ; c_{z}$ is the specific heat of liquid in the evaporator, $\mathrm{kJ} /\left(\mathrm{kg} \cdot{ }^{\circ} \mathrm{C}\right) ; T_{z}$ is the temperature of liquid in the evaporator Temperature, ${ }^{\circ} \mathrm{C} ; h_{i n, z}$ and $h_{o u t, z}$ are the specific enthalpy of working medium in and out of evaporator, $\mathrm{kJ} / \mathrm{kg} ; Q_{z}$ is the heat flux released by refrigerant water to medium in evaporator,KW.

\subsection{Model of heat exchanger and throttle valve}

For the heat exchanger, the heat conduction along the tube length of the heat exchanger is not considered, the flow change caused by the change of solution concentration and temperature is ignored, the specific heat and density change of the solution at the inlet and outlet of the heat exchanger are ignored, and the dynamic mathematical model of lithium bromide concentrated solution and dilute solution of the solution heat exchanger is established by using lumped parameters ${ }^{[8]}$ as shown in equation (10):

$$
\left\{\begin{array}{c}
c_{h x} M_{h x} \frac{d\left(\frac{T_{h x 1}+T_{h x 2}}{2}\right)}{d t}=Q_{h m x}-c_{h x} m_{h x}\left(T_{h x 2}-T_{h x 1}\right) \\
c_{h n} M_{h n} \frac{d\left(\frac{T_{h n 1}+T_{h n 2}}{2}\right)}{d t}=c_{h n} m_{h n}\left(T_{h n 1}-T_{h n 2}\right)-Q_{h m n} \\
c_{h m} M_{h m} \frac{d T_{h m}}{d t}=Q_{h m n}-Q_{h m x} \\
Q_{h m n}=k_{h n} A_{h}\left(\frac{T_{h n 1}+T_{h n 2}}{2}-T_{h m}\right) \\
Q_{h m x}=k_{h x} A_{h}\left(T_{h m}-\frac{T_{h x 1}+T_{h x 2}}{2}\right)
\end{array}\right.
$$

Where, $Q_{h m x}$ and $Q_{h m n}$ are the heat release power from heat exchanger plate to dilute solution and concentrated solution to heat exchanger plate, $\mathrm{KW} ; c_{h x}$ and $c_{h n}$ are the specific heat of solution at dilute solution side and concentrated solution side of heat exchanger, $\mathrm{kJ} /(\mathrm{kg} \cdot \mathrm{K}) ; M_{h x}$ and $M_{h n}$ are the solution mass at dilute solution side and concentrated solution side of heat exchanger, $\mathrm{kg} ; m_{h x}$ and $m_{h n}$ are the solution mass flow at dilute solution side and concentrated solution side of heat exchanger, $\mathrm{kg} / \mathrm{s} ; T_{h x l}$ and $T_{h \times 2}$ are the inlet and outlet temperatures of the dilute solution, ${ }^{\circ} \mathrm{C} ; T_{h n 1}$ and $T_{h n 2}$ are respectively the inlet and outlet temperatures of the concentrated solution, ${ }^{\circ} \mathrm{C} ; k_{h n}$ and $k_{h x}$ are respectively the heat transfer coefficient between concentrated solution/ dilute solution and heat exchanger plate, $A_{h}$ is the heat exchange area of the heat exchange 
plate, $\mathrm{m}^{2} ; T_{h m}$ is the metal temperature of the heat exchange plate, ${ }^{\circ} \mathrm{C}$.

Because of the short residence time of refrigerant in the throttle expansion valve, it is assumed that the throttle expansion process is an ideal adiabatic isoenthalpy process. Under the condition of a certain condensation temperature and evaporation temperature, the calculation formula of working fluid flow through the throttle expansion valve is shown in equation (11):

$$
m_{i n, z}=\psi \sqrt{2 \rho_{l, l}\left(p_{l}-p_{z}\right)}
$$

Where, $\psi$ is the flow coefficient; $\rho_{l, l}$ is the working medium density in front of the valve, $\mathrm{kg} / \mathrm{m}^{3} ; p_{l}$ and $p_{z}$ are the pressure in the condenser and evaporator respectively, $\mathrm{Pa}$.

\section{Model validation and test analysis}

All the models in this paper are developed based on MATLAB/Simulink platform, and the existing solvers of differential equations and partial differential equations are used to solve the models; the specific heat, density, specific enthalpy, dynamic viscosity, thermal conductivity and other physical property data of lithium bromide solution, liquid water and gaseous water vapor in the models are from the physical property regression equations in literature ${ }^{[9,10]}$.

\subsection{Model validation}

The data of $\mathrm{LiBr}$ absorption heat pump in reference ${ }^{[8]}$ is used to initialize the dynamic model, and the simulation results are compared with the design conditions, as shown in Table1; after the inlet temperature of refrigerant water is increased by $1{ }^{\circ} \mathrm{C}$, the dynamic changes of the data in reference $^{[8]}$ and the data calculated by this model are compared as shown in Fig2. Table1 shows that the steady-state results of the model established in this paper and the dynamic model established in the literature have a small error, which can accurately simulate the steady-state characteristics of the absorption heat pump. Fig2 shows that the model established in this paper can also accurately describe the dynamic characteristics of the absorption heat pump.

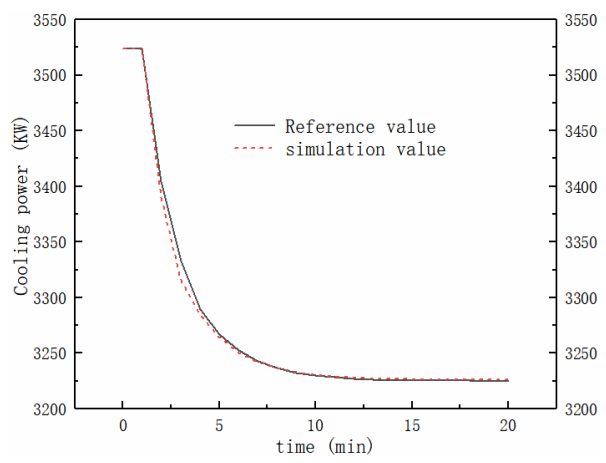

Fig 2 Comparison of dynamic changes of cooling capacity of heat pump

Tab 1 Data comparison table of absorption heat pump

\begin{tabular}{lccc}
\hline \multicolumn{1}{c}{ parameter } & literature $^{[\mathbf{8}]}$ & $\begin{array}{c}\text { Simulation } \\
\text { value }\end{array}$ & $\begin{array}{c}\text { Relative error } \\
(\mathbf{\%})\end{array}$ \\
\hline Heat load of generator $(\mathrm{kW})$ & 4861.6 & 4841.37 & 0.416 \\
Heat load of condenser $(\mathrm{kW})$ & 3743.6 & 3778.74 & 0.939 \\
Heat load of absorber $(\mathrm{kW})$ & 4620.7 & 4625.3 & 0.099 \\
Cooling power $(\mathrm{kW})$ & 3498.8 & 3514.79 & 0.457 \\
Evaporation pressure $(\mathrm{Pa})$ & 1030 & 1042 & 1.165 \\
Condensation pressure $(\mathrm{Pa})$ & 10000 & 10016 & 0.16 \\
Outlet temperature of refrigerant water $\left({ }^{\circ} \mathrm{C}\right)$ & 10.5 & 10.44 & 0.571 \\
Outlet temperature of cooling water $\left({ }^{\circ} \mathrm{C}\right)$ & 40 & 40.46 & 1.15 \\
COP & 0.72 & 0.729 & 1.25 \\
\hline
\end{tabular}

\subsection{Performance analysis of unit}

In order to explore the factors affecting the efficient operation of absorption heat pump, the absorption heat pump model is initialized according to the data in reference ${ }^{[8]}$. Under steady-state conditions, the change curves of cooling load and equipment COP(Coefficient Of Performance) caused by the change of inlet water temperature of driving heat source, inlet temperature of refrigerant water and inlet temperature of cooling water are shown in Fig 3-Fig5.

Fig3 shows that: with the increase of the inlet water temperature of the heat source, the refrigerant evaporation capacity increases, resulting in the increase of the cooling capacity. When the inlet temperature of the heat source further increases, the increase proportion of the cooling capacity is gradually less than the energy consumption required to increase the temperature of the heat source, resulting in the COP of the system first increases and then decreases, which provides a reference for the design of the inlet temperature range of the heat source. 


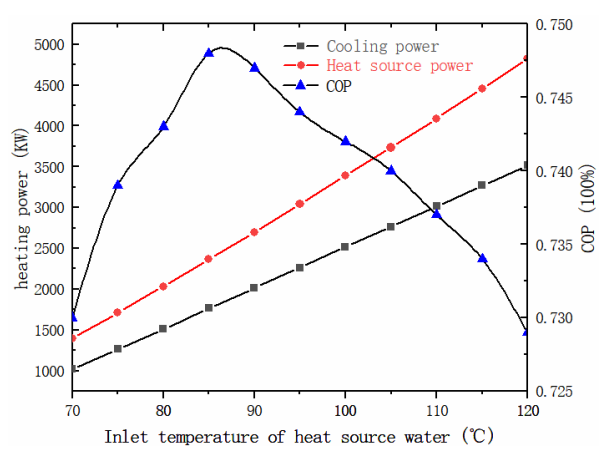

Fig3 Influence curve of heat source inlet temperature change on thermal performance

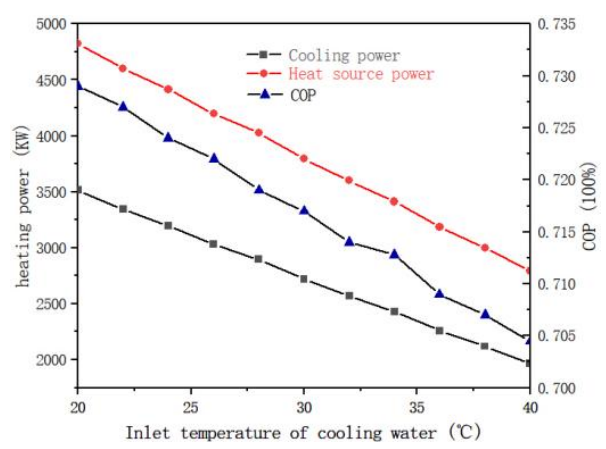

Fig4 Influence curve of inlet temperature change of cooling water on thermal performance

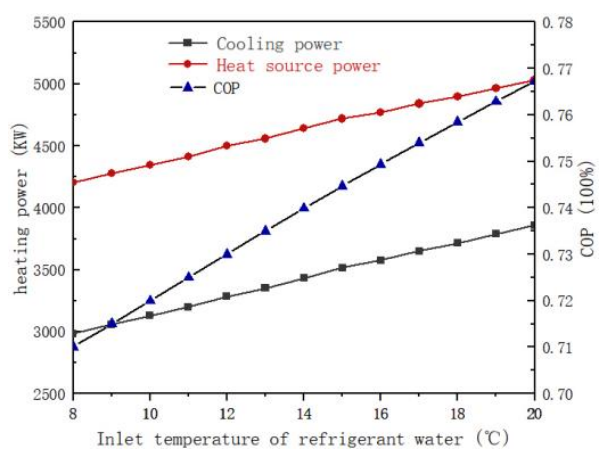

Fig5 Influence curve of inlet temperature change of refrigerant water on thermal performance

Fig4 shows that: the increase of cooling water temperature will cause the increase of evaporation temperature, which will increase the pressure of evaporator and generator, and reduce the heat exchange temperature difference of generator, thus reducing the evaporation capacity of refrigerant water, and finally causing the decrease of refrigeration power and COP of system, so the factors such as economy and seasonal change should be considered in the design.

Fig. 5 shows that : when the inlet temperature of the refrigerant water increases, the evaporation pressure increases, the mass fraction of the solution in the absorber increases, and the adsorption capacity becomes stronger, which leads to the increase of the refrigerating capacity and coefficient of performance. When the temperature of the refrigerant water increases to a certain extent, the outlet temperature of the refrigerant water will also increase correspondingly under the condition of a certain heat exchange temperature difference, which may lead to the failure to meet the needs of users, and it needs to be adjusted in the design considering the needs of users and the requirements of economy.

\subsection{Thermal inertia analysis of unit}

Under the steady-state operation condition of the absorption heat pump, when the inlet temperature of the driving heat source rises by $10{ }^{\circ} \mathrm{C}$ and the initial mass of the solution in each equipment is different, the dynamic change curves of the outlet temperature of the cooling water and the outlet temperature of the refrigerant water are shown in Fig.6-Fig7

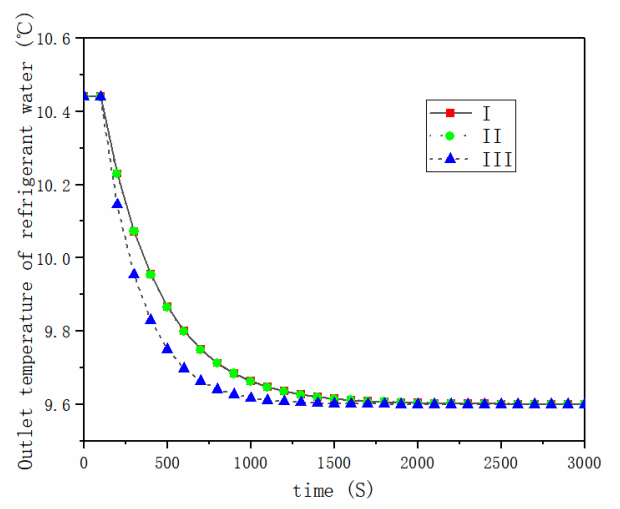

I-the mass of solution in each part is $8 \mathrm{~kg}$; II-the mass of solution in the absorber and generator is $8 \mathrm{~kg}$, the mass of solution in evaporator and condenser is $5 \mathrm{~kg}$; III-the mass of solution in each part is $5 \mathrm{~kg}$

Fig6 Dynamic change curve of outlet temperature of refrigerant water

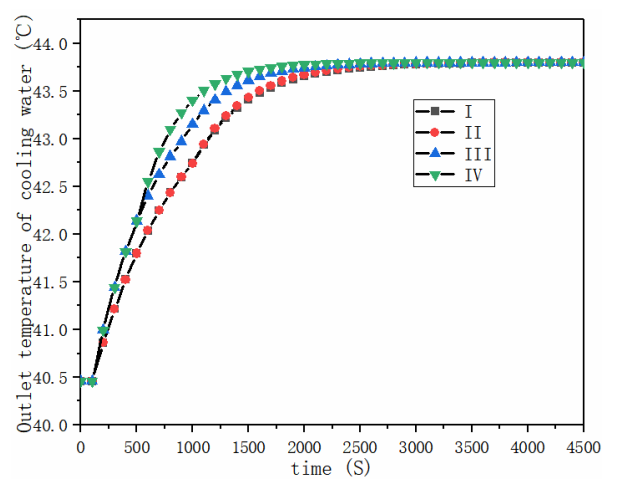

I - the mass of solution in each part is $8 \mathrm{~kg}$; II - the mass of solution in absorber and generator is $8 \mathrm{~kg}$, the mass of solution in evaporator and condenser is $5 \mathrm{~kg}$; III - the mass of solution in absorber is $8 \mathrm{~kg}$, and that in other parts is $5 \mathrm{~kg}$;

IV - the mass of solution in each part is $5 \mathrm{~kg}$

Fig7 Dynamic change curve of outlet temperature of cooling water

Fig6 and Fig7 show that: in the case of heat source disturbance, the dynamic response time of the outlet temperature of the cooling water increases with the increase of the mass of $\mathrm{LiBr}$ solution in the generator, 
and has no obvious relationship with the mass of the stock liquid in the evaporator and condenser; the dynamic response of the outlet temperature of the circulating cooling water is mainly affected by the thermal inertia of the generator in the early stage, and by the thermal inertia of the generator and the absorber in the later stage The superimposed influence of sex. This provides a reference for the dynamic control of cooling and heating load of absorption heat pump in operation.

\subsection{Analysis of crystallization characteristics}

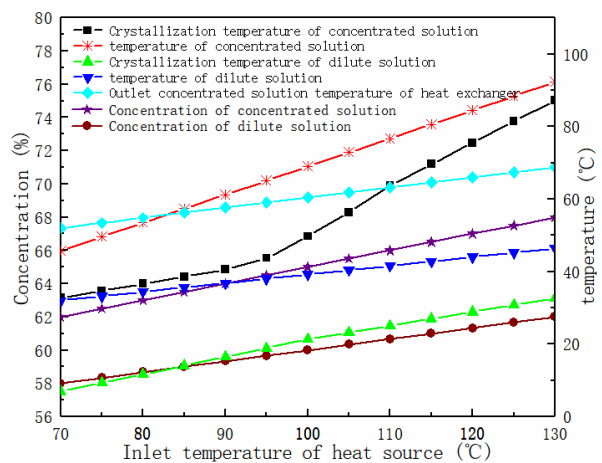

Fig 8 Influence curve of heat source inlet temperature on unit crystallization

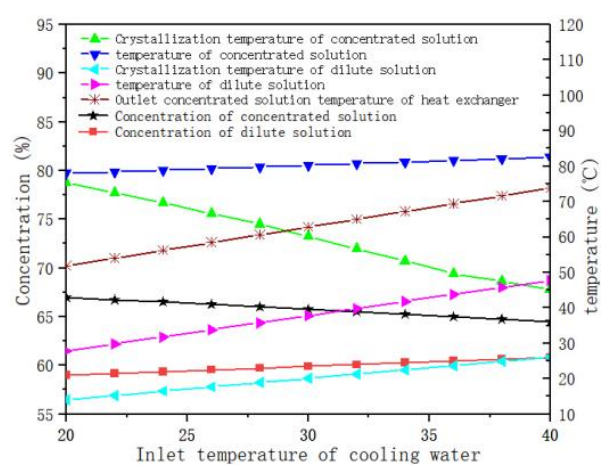

Fig 9 Influence curve of cooling water inlet temperature on unit crystallization

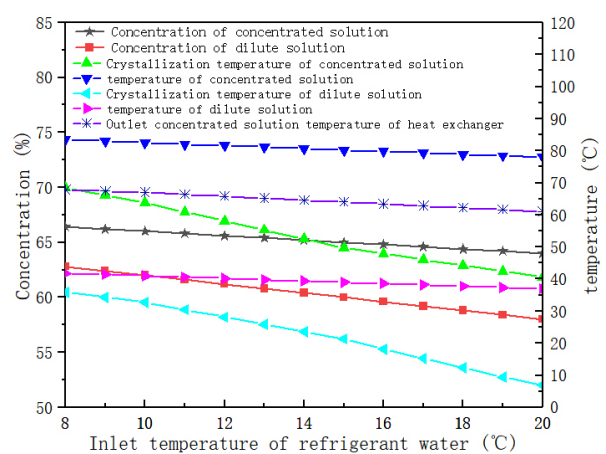

Fig 10 Influence curve of refrigerant water inlet temperature on unit crystallization

The crystallization of lithium bromide solution is related to the temperature and concentration of the solution. In order to explore the crystallization characteristics of absorption heat pump under various working conditions, the influence curves of heat source inlet temperature change, circulating cooling water inlet temperature change and refrigerant water inlet temperature change on the parameters of the unit under stable condition are tested, as shown in Fig8Fig10.

Fig 8 shows that: with the increase of heat source temperature, the concentrations of concentrated solution and dilute solution show an upward trend, and the concentration difference has an increasing trend. The crystallization temperature increases with the increase of concentration (crystallization curve of lithium bromide solution). Although the temperatures of concentrated solution and dilute solution increase with the increase of heat source temperature, the crystallization temperature margin of concentrated solution and dilute solution shows a decreasing trend, With the increase of heat source temperature, the solution has the risk of crystallization. When the concentration of concentrated solution reaches about $65 \%$, the crystallization has occurred at the outlet of concentrated solution in the heat exchanger. It can be seen from Fig9 that: with the decrease of cooling water inlet temperature, the concentration of dilute solution decreases, while the concentration of concentrated solution increases. Although the COP increases due to the increase of concentration difference, it also brings the risk of crystallization if the inlet temperature is too low, the concentrated solution will crystallize at the outlet of the heat exchanger; Fig 10 shows that: with the decrease of the inlet temperature of the refrigerant water, the concentration of the concentrated solution and the dilute solution presents an upward trend, and the concentration difference decreases, which leads to the decrease of COP, and the crystallization risk will occur at the outlet of the concentrated solution of the heat exchanger. If the inlet temperature of refrigerant water is too low, there may be the risk of equipment shutdown caused by icing in the refrigerant water pipe.

\section{5 conclusion}

In this paper, the dynamic model of absorption heat pump is established, the thermal inertia characteristics of the system are studied, and the performance characteristics and crystallization characteristics of the unit are analyzed under the changes of heat source inlet temperature, cooling water inlet temperature and refrigerant water inlet temperature:

1> The dynamic model can simulate not only the static condition of the system, but also the dynamic performance of the absorption heat pump system under dynamic disturbance.

$2>$ The dynamic response of the absorption heat pump is obviously affected by the solution quality of the generator and the absorber. The dynamic response of the refrigerant water is mainly affected by the thermal inertia of the solution quality of the generator, while the dynamic response of the outlet temperature of the circulating cooling water is comprehensively 
affected by the solution quality of the generator and the absorber.

$3>$ The increase of heat source inlet temperature makes the COP of the system increase first and then decrease. Too high heat source temperature will increase the crystallization risk of concentrated solution at the outlet of generator and heat exchanger. The decrease of cooling water inlet temperature will increase the COP of the system, but will also increase the crystallization risk of the system. The rise of refrigerant water temperature will increase the COP of the system, and will not bring crystallization risk, but will cause the outlet temperature can not meet the needs of users, which provides a good reference for enterprises in the parameter design stage and dynamic operation control of absorption heat pump.

\section{Reference}

1. Jeong S, Kang B, Karng S. (1998).Dynamic simulation of an absorption heat pump for recovering low grade waste heat[J]. Applied Thermal Engineering, 18(1): 1-12.

2. Kohlenbach P, Ziegler F. (2008)A dynamic simulation model for transient absorption chiller Performance. Part I: The model[J]. International Journal of Refrigeration, 31(2): 217-225.

3. Kohlenbach P, Ziegler.(2008)A dynamic simulation model for transient absorption chiller performance Part II: The model[J]. International Journal of Refrigeration,31(2): 217-225.

4. KHALID A J, ALI H L.(2001)Simulation of a simple absorption refrigeration system $[\mathrm{J}]$. Energy Conversion and Management, 42: $1575-1605$.

5. SUGANO N,SAITO M.(1996)Simulation and experimental research of start-up characteristics of single-effect absorption refrigerators driven by waste hot water $[\mathrm{J}]$. Transaction of JSME, 62(596): 304-310.

6. Iranmanesh A, Mehrabian M A.(2013)Dynamic simulation of a single-effect $\mathrm{LiBr}-\mathrm{H} 2 \mathrm{O}$ absorption refrigeration cycle considering the effects of thermal masses[J] . Energy and Buildings, 60: 47-59.

7. Auracher $\mathrm{H}$, Wohlfeil A, Ziegler F. (2008)A simple physical model for steam absorption into a falling film of aqueous lithium bromide solution on a horizontal tube[J]. Heat and Mass Transfer, 44(12): 1529-1536.

8. Le Hongtian. (2009)Modeling and dynamic simulation of $\mathrm{LiBr}$ absorption heat pump system [D]. North China Electric Power University (Beijing).

9. Florides G A, Kalogirou S A, Tassou S A, et al.(2003)Design and construction of a LiBr-water absorption machine[J]. Energy Conversion and Management, 44(15): 248-2508.

10. Jia Mingsheng. (2002)Calculation method of main physical parameters of lithium bromide aqueous solution [J]. Journal of Zhanjiang Ocean University, 22 (3): 52-58. 